\title{
Article
}

\section{A survey of the current practice of intramuscular Botulinum toxin injections for hemiplegic shoulder pain in the UK}

\author{
Holmes, Richard J and Connell, Louise \\ Available at http://clok.uclan.ac.uk/20780/ \\ Holmes, Richard J and Connell, Louise ORCID: 0000-0002-0629-2919 (2019) A \\ survey of the current practice of intramuscular Botulinum toxin injections for \\ hemiplegic shoulder pain in the UK. Disability and Rehabilitation, 41 (6). pp. \\ 720-726. ISSN 0963-8288
}

It is advisable to refer to the publisher's version if you intend to cite from the work. http://dx.doi.org/10.1080/09638288.2017.1400596

For more information about UCLan's research in this area go to http://www.uclan.ac.uk/researchgroups/ and search for < name of research Group>.

For information about Research generally at UCLan please go to http://www.uclan.ac.uk/research/

All outputs in CLoK are protected by Intellectual Property Rights law, including Copyright law. Copyright, IPR and Moral Rights for the works on this site are retained by the individual authors and/or other copyright owners. Terms and conditions for use of this material are defined in the policies page.

\section{CLoK}

Central Lancashire online Knowledge www.clok.uclan.ac.uk

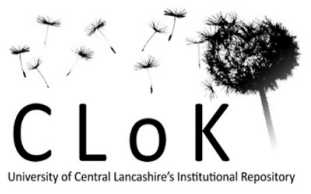




\title{
A Survey of the Current Practice of Intramuscular Botulinum Toxin Injections for Hemiplegic Shoulder Pain in the UK
}

\begin{abstract}
Purpose: To describe the current UK practice for the use of intramuscular Botulinum Toxin type A injections to treat hemiplegic shoulder pain.

Method: A UK-based cross-sectional study using an online survey. Participants ( $n=68)$ were medical and non-medical practitioners recruited via the membership of the British Society for Rehabilitation Medicine and the British Neurotoxin Network. Data was analysed using descriptive statistics and content analysis.

Results: The majority of respondents would consider Botulinum Toxin type A for hemiplegic shoulder pain $(86.8 \%)$, though most of these respondents inject for this goal infrequently (83.1\%). Pectoralis major was most commonly selected to achieve this goal. Barriers to this intervention included difficulties determining the cause of pain (29.4\%), difficulty isolating muscles (27.9\%), and a lack of evidence $(25 \%)$. The doses reported regularly deviated from guidelines and a substantial range in the volumes suggested was observed. Clinicians were mostly reliant on unstandardised measures to assess outcomes.

Conclusions: Current UK practice of Botulinum Toxin type A injections for hemiplegic shoulder pain associated with spasticity is highly variable. There are large gaps between current practice and available evidence with regards to muscle selection and doses used. A number of areas for further investigation have been identified to progress current understanding of this intervention.
\end{abstract}

Keywords: Botulinum toxin; hemiplegic shoulder pain; post-stroke shoulder pain; spasticity; stroke; survey; 


\section{Background}

Hemiplegic shoulder pain (HSP) is a common complication following stroke ranging in incidence between $23 \%$ [1] to $40 \%$ [2] at six months, with a peak onset of four months [3]. It is associated with an increased length of stay during inpatient rehabilitation and is an important predictor of poor functional outcome in the upper limb [4,5]. It has been shown to be significantly associated with reduced quality of life at 12 months post-stroke [6], as well as impacting on a patient's mood, activities of daily living and participation in leisure activities to a great extent [7].

HSP is thought to be multifactorial in causation, encompassing both neurological (of central or peripheral nature) and mechanical (traumatic and insidious) factors [8]. Consequently, the range of available treatment modalities is vast with one exploratory study identifying 175 different therapeutic interventions under 13 themes [9]. Roosink et al [10] recommend a multifactorial approach to treatment but to do this there needs to be a clear diagnostic process to identify the different aspects of HSP at play. Unfortunately, it remains unclear in the literature what the optimal treatment modalities for the various subtypes of HSP are and, in practice, linking the causation with the most effective intervention remains problematic.

Studies have found a high correlation between HSP and decreased range of passive shoulder movement [11,12], but it is unclear if this is a causative relationship and in practice it is difficult to distinguish the degree to which different factors (such as contracture, spasticity, or adhesive capsulitis) contribute to the restriction and pain [13]. A number of randomised controlled studies have hypothesised that HSP associated with range-limiting spasticity around the shoulder girdle may improve with intramuscular Botulinum Toxin type A (BoNT-A) injections [14-19]. However, these studies found conflicting results likely secondary to the 
variability in doses, dilutions and products used as well as the range of muscles selected to treat. They also lacked attention to activity-based or goal-orientated outcome measures. Because of this variability in methodology, definitive recommendations to achieve the optimal effectiveness of this treatment modality are not currently possible.

\section{A Cochrane review [20] and a meta-analysis [21] reviewed BoNT-A injections for HSP} and both found the evidence favoured the intervention. However, the few studies they reviewed were limited by low powered randomised controlled trials and a high potential for bias. The recent Royal College of Physicians' guidelines for stroke [22] also comment that whilst BoNT-A injections showed positive benefits in reducing pain and improving function and range of movement, there remains a need for larger, high-quality randomised controlled trials before the intervention can be confidently recommended in routine clinical practice.

In the future, these larger-scale studies will need to explore the factors that contribute to optimising effective patient selection, muscle selection and dosage. However, prior to this it would be useful to understand the current 'state of play' to see if there is a consensus in the application of this intervention in practice and to identify any gaps between the available evidence and contemporary practice. With this in mind, an online survey was undertaken with the aim of ascertaining the current practice for the use of intramuscular BoNT-A injections to treat HSP in the UK and to compare this to existing guidelines. 


\section{Method}

\section{Study design}

A UK-based cross-sectional study design was used with data collected via an online survey. Ethical approval was granted by the University of Central Lancashire Research Ethics Committee (reference number STEMH 595).

\section{Survey design}

A review was carried out to identify any valid and reliable tools within the literature. As none were identified, an original survey was designed using the online survey tool, SurveyMonkey®. The survey consisted of three sections - (i) Demographics, (ii) Assessment and Treatment, and (iii) Factors Affecting Clinical Decision Making. The questionnaire utilised a mix of multiple-choice, Likert scales and open-ended questions. A question skip logic was applied whereby if participants would not consider using BoNT-A for HSP associated with spasticity then they skipped questions related to the methods used for such injections.

During the development and refining stage, the survey was initially trialled on a group of eight professionals including two doctors and six physiotherapists. These were a mix of professionals with a knowledge of the topic matter $(n=3)$, a knowledge of research methods $(n=2)$, and "lay-persons" with no specific expertise in either $(n=3)$. A second round of testing $(n=4)$ was undertaken to assess usability and for further revisions to be made to the questionnaire. Prior to distribution the survey was peer reviewed by the Research and Clinical Standards Committee at the British Society for Rehabilitation Medicine, following which the structure and content of the survey was finalised (Supplemental online material). 


\section{Participants}

The sample population for this study was medical and non-medical practitioners in the UK who inject BoNT-A for spasticity management as part of their current practice. The British Society for Rehabilitation Medicine and the British Neurotoxin Network distributed the link to the online survey to their members via email. The email contained the participant information sheet as an attachment and recipients were asked to forward the link on to other practitioners as appropriate, thus sourcing additional participants via snowball sampling. A second email was distributed two weeks later to act as a reminder.

Participants were advised that completion of the survey would be taken as their informed consent. The use of the online survey tool ensured participants anonymity remained intact. No incentives were offered for involvement in the study.

\section{Data analysis}

Data was collected and securely stored on the SurveyMonkey® website. Data was then downloaded into an Excel format and imported into SPSS for analysis. Descriptive statistics were used to describe, synthesise, and summarise the data. Content analysis was used to collate and report the responses from open-ended responses.

During analysis, Likert scales were recoded to group together positive, neutral and negative responses. Doses were also recoded as under, over or within the recommended dose range when compared to the RCP National Guidelines: Spasticity in Adults [23]. As these guidelines do not provide recommended doses for Xeomin ${ }^{\circledR}$, a conversion ratio of $1: 1$ (Botox®: Xeomin ${ }^{\circledR}$ ) was used as described by Dressler et al [24]. Due to low frequencies of most muscles reported, only the five most commonly suggested muscles had sufficient data to analyse the doses and volumes used. 


\section{Results}

\section{Participant demographics}

The survey link was distributed to 217 members of the British Neurotoxin Network and 305 members of the British Society for Rehabilitation Medicine, giving a total of 522 recipients. Although it was not possible to identify the exact number of appropriate practitioners who received the link, a total of 93 participants responded giving an approximate initial response rate of $17.8 \%$. Of these, 25 respondents submitted incomplete questionnaires and were therefore excluded from the study. Consequently, 68 completed questionnaires were included in the final analysis giving an actual response rate of $13.0 \%$. The demographic characteristics of these participants can be seen in Table 1.

All areas of the UK were represented within the data (Figure 1). Practitioners had been qualified in the range between five to 46 years (mean=22.57 years, $\mathrm{SD}=9.23$ years). Their highest academic qualifications were grouped into graduate (35.3\%), post-graduate $(27.9 \%)$, and doctoral (26.5\%) levels with some respondents citing higher medical qualifications (FRCP 5.9\%, MRCP 2.9\%, FRCSI 1.5\%). Over half (54.4\%) had been injecting BoNT-A for over 10 years and approximately a third (33.8\%) performed over 20 injections per month (Table 1). Respondents reported injecting BoNT-A in a range of settings including inpatient acute wards (55.9\%), inpatient rehabilitation wards (77.9\%), outpatient clinics (88.2\%), and on domiciliary visits $(42.6 \%)$.

\section{Clinician's use and experience}

The majority of respondents reported that they would consider injecting intramuscular BoNTA for HSP associated with spasticity (86.8\%). Due to limited numbers across demographic factors it was not possible to calculate any statistical differences between groups. Just over 
half $(54.4 \%)$ agreed or strongly agreed that BoNT-A is an effective treatment for HSP. However, it is often not their first-line treatment choice with only $8.8 \%$ agreeing or strongly agreeing to this effect.

Despite the high number of respondents who would inject, only $45.6 \%$ reported they had no barriers to injecting BoNT-A for HSP. The main barriers that respondents reported were difficulties determining the cause of pain (29.4\%), difficulty isolating the muscles to inject (27.9\%), and a lack of evidence (25\%) (Table 2).

Of the clinicians who would consider injections $(n=59)$ the majority $(83.1 \%)$ had only performed three or less injections for the goal of reducing HSP in the last three months. When deciding on the timings of injections post-stroke, the majority of clinicians would consider injecting at the stages ' $1-3$ months' and ' $3-6$ months' (83.1\%). This is in contrast to the ' $0-13$ days' stage where only $11.9 \%$ of clinicians would consider injecting BoNT-A for HSP.

With regards to signs and symptoms that would lead respondents to believe that HSP was due to spasticity over other causes, the most common reported were 'Limitation of shoulder abduction' (71.2\%), 'Limitation of shoulder external rotation' (69.5\%), 'Flexor patterning in the upper limb' (61.0\%), and 'Pain on passive movement' (54.2\%) (Table 3).

Content analysis found few adverse effects with four respondents reporting the usual side effects of localised pain and bruising at the injection site, four respondents reporting increased/worsening subluxation, and one respondent reporting that the shoulder was more painful following injections if not supported with a brace. There was only one report of a serious adverse event in which a patient suffered dysphagia following injections that included muscles around the shoulder. However, it was unclear which muscles were injected and whether this was done for the goal of reducing HSP. In support of the low number of reported problems, only $4.4 \%$ agreed or strongly agreed that BoNT-A injections for HSP cause adverse effects. 


\section{Current practice}

The primary muscle that clinicians chose to inject most commonly was pectoralis major, being the first choice of $67.8 \%$ of the questioned cohort $(n=59)$. Other muscles considered as first choice were subscapularis (10.2\%), supraspinatus (5.1\%), biceps brachii (1.7\%), teres major $(1.7 \%)$, and infraspinatus $(1.7 \%)$. When collating all responses, including second and third choices, there was a wide range of muscles suggested and the frequencies for each can be seen in Table 4.

There was a large degree of variation observed with regards to the doses and volumes of injectate used. The most common muscle identified (pectoralis major) was reported to be injected below the recommended dose by just under half of respondents, whilst for other muscles (subscapularis and teres major) there was a trend towards higher than recommended doses. The volumes of injectate used also showed a substantial degree of variability for all muscles analysed. This was most strikingly apparent for pectoralis major, subscapularis and latissimus dorsi whereby the maximum volume recommended by respondents was 10 to 16times greater than the minimum volume. Data for the doses and volumes of the five most common muscles is summarised in Table 5.

Content analysis of how clinicians assess the effectiveness of injections for HSP found that only $33.9 \%$ of respondents referred to a standardised outcome measure. The most common outcome measures reported were visual analogue scale $(22.0 \%, n=13)$, and goal attainment scaling $(11.9 \%, n=7)$. However, more often clinicians referred to the use of unstandardised subjective and objective assessment with $27.1 \%(n=16)$ of respondents citing patient, carer or therapist reports, and $16.9 \%(\mathrm{n}=10)$ citing clinician subjective review. 


\section{Discussion}

The main finding of this study was the wide variation in practice reported for the goal of treating spasticity associated HSP with BoNT-A. This is in support of the Upper Limb International Spasticity (ULIS)-II study [25] who also described a wide variation in practice in relation to treatment goals for the upper limb. However, whereas ULIS-II reported BoNT-A treatment for upper limb spasticity in general, this study focussed specifically on a single goal of intervention thereby making the observation even more unexpected.

Whilst it is acknowledged that the complexities of spasticity as well as HSP demand an individualised treatment intervention [26], it was not expected that practice would be so widely varied with regards to muscle selection, dose, and volume as well as the outcome measures used. Baguley et al [27] explored the decision-making practices with regards to BoNT-A use for adult upper limb spasticity and found that the muscle selection and doses used were not significantly associated with the goals of treatment or the severity of spasticity. They concluded that injector beliefs more so than patient characteristics were the main force guiding clinical decision making. Further exploration of the factors influencing this variation is warranted.

The majority of respondents would consider injections for HSP, but only $54.4 \%$ thought it was effective. The reasons for this remain unclear, but may emphasise difficulties clinicians have in relation to clinical reasoning for this intervention. Clinicians reported they injected for HSP very infrequently with $83 \%$ having done three or less injections in the last three months. This may be related to the main barriers to injecting found in this study. If clinicians have difficulty determining the cause of HSP then there will undoubtedly be uncertainty in the management of the pain. This finding compliments the work of Bakheit et al [28] who in an 
international survey found an infrequency of shoulder injections in practice and postulated that this could be due to the complex and multifactorial nature of HSP.

Interestingly, in this study, pectoralis major was overwhelmingly the muscle most often selected to treat HSP. Yet in the literature, pectoralis major and subscapularis have been investigated equivalently. This disparity between research and clinical practice could be speculated to be due to several reasons including the difficulty of injecting subscapularis and the potential need for imaging. However, if a restriction of shoulder external rotation is associated with the development of HSP [29] then subscapularis should be given more consideration in clinical practice given that it is the main force producer of internal rotation at the shoulder [30].

The BoNT-A doses that were reported in this study deviated notably from the RCP guidelines for spasticity management [23]. Franke et al [31] report that more experienced clinicians may be less likely to follow clinical guidelines than their less experienced counterparts. The large number of experienced clinicians in this study could therefore account for this deviation. Interestingly, studies $[14,17]$ have found a positive effect of BoNT-A on HSP when they used approximately double the dose of that suggested in guidelines, suggesting that the larger doses suggested in this study may indeed be warranted to achieve an analgesic effect in HSP.

This gap between evidence and clinical practice is a common finding in many areas and is well documented in the literature $[32,33]$. There are many barriers to changing practice in response to guidelines reported in the literature [34]. Whilst an understanding of the specific barriers to evidence-based practice is thought to be a crucial first-step to bridge the gap between evidence and practice, it is still not understood what the most effective strategies to achieve this change are [35]. To this end, an initial exploration of the specific factors that impact on 
the uptake of clinical guidelines in spasticity management would be useful, particularly on the matter of dosing.

As previously discussed, the literature reports difficulties in determining causation of HSP due to the multifactorial nature of the condition [8]. Understanding the factors that would help a clinician identify which patients would respond to this intervention is paramount to optimise the effectiveness of BoNT-A injections. Through consensus of opinion, this study has identified that clinicians feel limitation of shoulder abduction and external rotation, flexor patterning of the upper limb, and pain on passive movement are the symptoms that would lead them to decide the HSP was due to spasticity over other causes. Further studies are required to investigate whether these symptoms effectively identify those patients who would most benefit from BoNT-A injections for HSP.

With regards to the timing of intervention, the survey showed that only $11.9 \%$ would consider injections within the first two weeks, which increased to $40.1 \%$ between two and four weeks. This is in comparison to the $83.1 \%$ who would consider injections between one to six months. This finding could be due to a concern that using BoNT-A injections early may inhibit motor recovery and clinicians may prefer to use a 'wait and see' approach at that point. It could also be that, when completing the survey, respondents were mindful of the typical initial post-stroke stage of flaccid paralysis which would negate the need for BoNT-A. However, in observational studies, spasticity was present in $21 \%$ [36] to $24.5 \%$ [37] of patients within the first two weeks post-stroke. Ward [38] comments that early intervention with BoNT-A may reduce severity, delay the progression, or even prevent the onset of spasticity after stroke. Therefore, if early identification and treatment of HSP associated with spasticity could be achieved then outcomes may be improved. 


\section{Limitations}

Limitations of this study include the inherent biases that can occur with an online survey of this nature. There may have been a degree of self-selection bias as clinicians with a particular interest in the topic area may be more likely to opt in to the study, leading to under-coverage of the targeted sample [39]. It is also possible that there may have been a degree of social desirability bias if subjects reported what they felt was expected practice rather than their actual routine practice $[40]$.

The response rate was low but this was not unexpected for a survey of this type where the subject matter is such a focussed specialism [41]. The use of British Society for

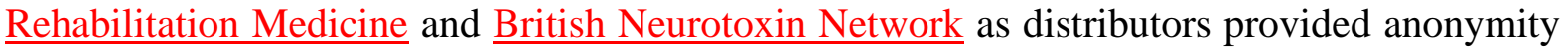
and credibility whilst targeting clinicians most likely to be involved in spasticity management. Efforts were made to optimise the response rate by developing a short, relevant survey and through the use of reminder emails [42]. Nonetheless, certain areas of the UK had only one or two respondents. Without an understanding of the location and number of injectors in the UK it is unclear if this is an under-representation of certain areas or due to a reduced response rate in general. In actuality, the overall response rate is likely to be higher than reported as the calculation used did not allow for invalid or inactive email addresses or respondents with multiple addresses in the database.

Finally, whilst every effort was made to ensure the clarity and readability of the survey through numerous reviews and refinement, there is the possibility that participants may have misunderstood the phrasing of questions [42]. In turn this could have led to frustration and explain the desire of a number of subjects to leave the survey before completion [43]. 


\section{Further Research}

Observational studies would be beneficial to better describe this complex intervention and to determine the conditions in which the intervention works most effectively [44]. This would identify areas of focus that could then be explored using experimental designs with comparative methods. Factors such as muscle selection, dose, and volume of injectate need to be more thoroughly explored to aid clinical reasoning for this intervention. It would also be beneficial to identify which patients are most likely to gain from this treatment to optimise the success rate. In the future, longitudinal studies investigating the timing of the intervention post-stroke to assess whether early BoNT-A injections could have a prophylactic effect on HSP would be worthwhile.

\section{Conclusion}

Current UK practice of BoNT-A injections for HSP associated with spasticity is highly variable. The causes for this remain unclear. There are also gaps between contemporary practice and the available evidence and guidelines, most notably in relation to muscle selection and the doses used. Further research is required to ascertain the most effective treatment approach and for optimal patient selection. An understanding of the early clinical signs of patients at risk of developing HSP associated with spasticity warrants further exploration as early intervention may reduce or prevent the complications associated with HSP.

\section{Acknowledgements}

The British Society for Rehabilitation Medicine and the British Neurotoxin Network are gratefully acknowledged for assisting with the dissemination of the survey to their membership groups, as are the clinicians who took the time to participate in the study. 


\section{Declaration of Interest}

The authors report no conflicts of interest. 


\section{References}

1. Ratnasabapathy Y, Broad J, Baskett J, et al. Shoulder pain in people with a stroke: a population-based study. Clin Rehabil. 2003;17:304-311.

2. Gamble GE, Barberan E, Laasch H-U, et al. Poststroke shoulder pain: a prospective study of the association and risk factors in 152 patients from a consecutive cohort of 205 patients presenting with stroke. Eur J Pain. 2002;6:467-474.

3. Adey-Wakeling Z, Arima H, Crotty M, et al. Incidence and Associations of Hemiplegic Shoulder Pain Poststroke: Prospective Population-Based Study. Arch Phys Med Rehabil. 2015;96:241-247.

4. Roy CW, Sands MR, Hill LD. Shoulder pain in acutely admitted hemiplegics. Clin Rehabil. 1994;8:334-340.

5. Barlak A, Unsal S, Kaya K, et al. Poststroke shoulder pain in Turkish stroke patients: relationship with clinical factors and functional outcomes. Int $\mathbf{J}$ Rehabil Res. 2009;32:309-315.

6. Adey-Wakeling Z, Liu E, Crotty M, et al. Hemiplegic Shoulder Pain Reduces Quality of Life After Acute Stroke. Am J Phys Med Rehabil. 2016;95:758-763.

7. Lindgren I, Gard G, Brogårdh C. Shoulder pain after stroke - experiences, consequences in daily life and effects of interventions: a qualitative study. Disabil Rehabil. 2017;[7 p]. DOI:10.1080/09638288.2017.1290699

8. Vasudevan JM, Browne BJ. Hemiplegic Shoulder Pain: An Approach to Diagnosis and Management. Phys Med Rehabil Clin N Am. 2014;25:411-437.

9. Pomeroy VM, Niven DS, Barrow S, et al. Unpacking the black box of nursing and therapy practice for post-stroke shoulder pain: a precursor to evaluation. Clin Rehabil. 2001;15:67-83. 
10. Roosink M, Renzenbrink GJ, Buitenweg JR, et al. Persistent Shoulder Pain in the First 6 Months After Stroke: Results of a Prospective Cohort Study. Arch Phys Med Rehabil. 2011;92:1139-1145.

11. Lindgren I, Lexell J, Jönsson A-C, et al. Left-Sided Hemiparesis, Pain Frequency, and Decreased Passive Shoulder Range of Abduction Are Predictors of Long-Lasting Poststroke Shoulder Pain. PM R. 2012;4:561-568.

12. Blennerhassett JM, Gyngell K, Crean R. Reduced active control and passive range at the shoulder increase risk of shoulder pain during inpatient rehabilitation post-stroke: an observational study. J Physiother. 2010;56:195-199.

13. Wilson RD, Chae J. Hemiplegic Shoulder Pain. Phys Med Rehabil Clin N Am. 2015;26:641-655

14. Yelnik AP, Colle FM, Bonan IV, et al. Treatment of shoulder pain in spastic hemiplegia by reducing spasticity of the subscapular muscle: a randomised, double blind, placebo controlled study of botulinum toxin A. J Neurol Neurosurg Psychiatry. 2007;78:845848.

15. De Boer KS, Arwert HJ, de Groot JH, et al. Shoulder pain and external rotation in spastic hemiplegia do not improve by injection of botulinum toxin A into the subscapular muscle. J Neurol Neurosurg Psychiatry. 2008;79:581-583.

16. Kong K-H, Neo J-J, Chua KSG. A randomized controlled study of botulinum toxin A in the treatment of hemiplegic shoulder pain associated with spasticity. Clin Rehabil. $2007 ; 21: 28-35$.

17. Marco E, Duarte E, Vila J, et al. Is Botulinum Toxin Type A Effective in the Treatment of Spastic Shoulder Pain in Patients After Stroke? A Double-Blind Rondomized Clinical Trial. J Rehabil Med. 2007;39:440-447. 
18. Lim J-Y, Koh J-H, Paik N-J. Intramuscular Botulinum Toxin-A Reduces Hemiplegic Shoulder Pain: A Randomized, Double-Blind, Comparative Study Versus Intraarticular Triamcinolone Acetonide. Stroke. 2008;39:126-131.

19. Marciniak CM, Harvey RL, Gagnon CM. Does botulinum toxin type A decrease pain and lessen disability in hemiplegic survivors of stroke with shoulder pain and spasticity?: a randomized, double-blind, placebo-controlled trial. Am J Phys Med Rehabil. 2012;91:1007-1019.

20. Singh JA, Fitzgerald PM. Botulinum toxin for shoulder pain. Cochrane Database of Systematic Reviews. 2010;[16 p]. DOI:10.1002/14651858.CD008271.pub2

21. Wu T, Fu Y, Song H, et al. Effectiveness of Botulinum Toxin for Shoulder Pain Treatment: A Systematic Review and Meta-Analysis. Arch Phys Med Rehabil. 2015;96:2214-2220.

22. Royal College of Physicians. National clinical guideline for stroke. Fifth Edition. London:RCP;2016.

23. Royal College of Physicians, British Society of Rehabilitation Medicine, Chartered Society of Physiotherapy, et al. Spasticity in adults: management using botulinum toxin. National Guidelines. London:RCP;2009.

24. Dressler D, Mander G, Fink K. Measuring the potency labelling of onabotulinumtoxinA (Botox®) and incobotulinumtoxinA (Xeomin $\left.{ }^{\circledR}\right)$ in an LD50 assay. J Neural Transm. 2012;119:13-15.

25. Turner-Stokes L, Fheodoroff K, Jacinto J, et al. Results from the Upper Limb International Spasticity Study-II (ULIS-II): a large, international, prospective cohort study investigating practice and goal attainment following treatment with botulinum toxin A in real-life clinical management. BMJ Open. 2013;3:e02771. DOI:10.1136/bmjopen-2013-002771 
26. Sheean G, Lannin NA, Turner-Stokes L, et al. Botulinum toxin assessment, intervention and after-care for upper limb hypertonicity in adults: international consensus statement. Eur J Neurol. 2010;17(Suppl.2):74-93.

27. Baguley IJ, Nott MT, Turner-Stokes L, et al. Investigatin Muscle Selection for Botulinum Toxin-A Injections in Adults with Post-Stroke Upper Limb Spasticity. J Rehabil Med. 2011;43:1032-1037.

28. Bakheit AM, Zakine B, Maisonobe $\mathrm{P}$, et al. The profile of patients and current practice of treatment of upper limb muscle spasticity with botulinum toxin type A: an international survey. Int J Rehabil Res. 2010;33:199-204.

29. Zorowitz RD, Hughes MB, Idank D, et al. Shoulder Pain and Subluxation After Stroke: Correlation or Coincidence? Am J Occup Ther. 1996;50:194-201.

30. ChangY-W, Hughes RE, Su F-C, et al. Prediction of muscle force involved in shoulder internal rotation. J Shoulder Elbow Surg. 2000;9:188-195.

31. Francke AL, Smit MC, de Veer AJE, et al. Factors influencing the implementation of clinical guidelines for health care professionals: A systematic meta-review. BMC Med Inform Decis Mak. 2008;8:38[p 11]. DOI:10.1186/1472-6947-8-38

32. Boaz A, Baeza J, Fraser A, et al. Effective implementation of research into practice: an overview of systematic reviews of the health literature. BMC Res Notes. 2011;4:212[p8]. DOI:10.1186/1756-0500-4-212

33. Grol R, Grimshaw J. From best evidence to best practice: effective implementation of change in patients' care. Lancet. 2003;362:1225-1230.

34. Sadeghi-Bazargani H, Tabrizi JS, Azami-Aghdash S. Barriers to evidence-based medicine: a systematic review. J Eval Clin Pract. 2014;20:793-802.

35. Grol R, Wensing M. What drives change? Barriers to and incentives for achieving evidence-based practice. Med J Aust. 2004;180:S57-S60. 
36. Sommerfield DK, Eek EU-B, Svensson A-K, et al. Spasticity After Stroke: Its Occurrence and Association With Motor Impairments and Activity Limitations. Stroke. 2004;35:134-140.

37. Wissel J, Schelosky LD, Scott J, et al. Early development of spasticity following stroke: a prospective, observational trial. J Neurol. 2010;257:1067-1072.

38. Ward AB. A literature review of the pathophysiology and onset of post-stroke spasticity. Eur J Neurol. 2012;19:21-27.

39. Bethlehem JG. Selection bias in web surveys. Int Stat Rev. 2010;78:161-188.

40. Krumpal I. Determinants of social desirability bias in sensitive surveys: a literature review. Qual Quant. 2013;47:2025-2047.

41. Fan W, Yan Z. Factors affecting response rates of the web survey: A systematic review. Computers in Human Behavior. 2010;26:132-139.

42. Evans JR, Mathur A. The value of online surveys. International Research. 2005;15:195219.

43. Ray NM, Tabor SW. Cyber Surveys come of age. Marketing Research. 2003;Spring:32-37.

44. Craig P, Dieppe P, Macintyre S, et al. Developing and evaluating complex interventions: the new Medical Research Council guidance. BMJ. 2008;337:979-983. 


\section{Figures \& Tables}

Figure 1. Respondents \% per UK region $(n=68)$

Table 1. Demographic characteristics $(n=68)$

\begin{tabular}{|rll|}
\hline & & $\%(n)$ \\
Profession & Medical & $73.5(50)$ \\
& Physiotherapy & $25.0(17)$ \\
& Nursing & $1.5(1)$ \\
Number of years & & \\
injecting BoNT-A & $0-2$ & $7.4(5)$ \\
& $3-5$ & $20.6(14)$ \\
& $6-10$ & $17.6(12)$ \\
& Over 10 & $54.4(37)$ \\
Frequency of BoNT-A & & \\
injections per month & $0-5$ & $14.7(10)$ \\
& $6-10$ & $25.0(17)$ \\
& $11-15$ & $16.2(11)$ \\
& $16-20$ & $10.3(7)$ \\
& Over 20 & $33.8(23)$ \\
\hline
\end{tabular}

Table 2. Barriers to BoNT-A injections for HSP $(n=68)$

\begin{tabular}{|rl|}
\hline Barriers & $\%(n)$ \\
None - I have no barriers to this & $45.6(31)$ \\
Difficulty determining cause of pain & $29.4(20)$ \\
Difficult to isolate muscles for injection & $27.9(19)$ \\
Lack of evidence & $25.0(17)$ \\
Other treatment options are more effective & $16.2(11)$ \\
Uncertain of muscle anatomy & $10.3(7)$ \\
Unable to access required equipment & $5.9(4)$ \\
Difficulty in obtaining funding for toxin or service & $5.9(4)$ \\
Need for imaging when injecting subscapularis & $4.4(3)$ \\
Delays with referrals & $2.9(2)$ \\
Concerns regarding safety & $1.5(1)$ \\
& \\
\hline
\end{tabular}


Table 3. Respondents use of BoNT-A injections for HSP $(n=59)$

Number of injections for HSP in last 3 months

$\%(n)$

Post-stroke stages where

BoNT-A considered for HSP

Signs and symptoms that HSP is due to spasticity

Limitation of shoulder abduction

$\begin{array}{ll}0 & 22.0(13)\end{array}$

$1-3 \quad 61.0(36)$

4-6 $8.5(5)$

Over $10 \quad 8.5(5)$

\section{$0-13$ days \\ $11.9(7)$ \\ 2-4 weeks \\ $40.1(24)$ \\ 1-3 months \\ $83.1(49)$ \\ 3-6 months 83.1 (49) \\ 6-12 months 66.1 (39) \\ Over 1 year \\ $62.7(37)$}

Limitation of shoulder external rotation

$71.2(42)$

Flexor patterning in the upper limb

Pain on passive movement

Malalignment

$69.5(41)$

$61.0(36)$

$54.2(32)$

$39.0(23)$

Pain on active movement 25.4 (15)

Pain on palpation of muscle 22.0 (13)

Pain described as dull ache $16.9(10)$

Pain described as sharp shooting 11.9(7)

Night pain $10.2(6)$

Pain at rest $8.5(5)$

Table 4. All muscles reported that respondents would consider for BoNT-A injections ( $n=59)$

\begin{tabular}{|rl|}
\hline Muscle & $\%(n)$ \\
Pectoralis major & $83.1(49)$ \\
Subscapularis & $28.9(17)$ \\
Biceps brachii & $16.9(10)$ \\
Teres major & $16.9(10)$ \\
Latissimus dorsi & $16.9(10)$ \\
Trapezius & $13.6(8)$ \\
Supraspinatus & $6.8(4)$ \\
Infraspinatus & $3.4(2)$ \\
Levator scapulae & $3.4(2)$ \\
Deltoid & $3.4(2)$ \\
Rhomboids & $1.7(1)$ \\
Triceps brachii & $1.7(1)$ \\
Pectoralis minor & $1.7(1)$ \\
Brachialis & $1.7(1)$ \\
Brachioradialis & $1.7(1)$ \\
\hline
\end{tabular}


Table 5. Doses and volumes of injectate of 5 most common muscles

\begin{tabular}{|l|l|l|l|l|l|l|}
\hline \multicolumn{2}{|c|}{} & $\begin{array}{l}\text { Pectoralis } \\
\text { major } \\
(n=46)\end{array}$ & $\begin{array}{l}\text { Subscapularis } \\
(n=17)\end{array}$ & $\begin{array}{l}\text { Biceps } \\
\text { brachii } \\
(n=10)\end{array}$ & $\begin{array}{l}\text { Teres } \\
\text { major } \\
(n=10)\end{array}$ & $\begin{array}{l}\text { Latissimus } \\
\text { dorsi } \\
(n=10)\end{array}$ \\
\hline $\begin{array}{l}\text { Dose } \\
\text { comparison } \\
(\%)\end{array}$ & $\begin{array}{l}\text { Under } \\
\text { recommended } \\
\text { dose }\end{array}$ & 47.8 & 11.8 & 20.0 & 0.0 & 20.0 \\
\cline { 2 - 7 } & $\begin{array}{l}\text { Within } \\
\text { recommended } \\
\text { dose }\end{array}$ & 30.4 & 23.5 & 70.0 & 20.0 & 30.0 \\
\cline { 2 - 7 } & $\begin{array}{l}\text { Over } \\
\text { recommended } \\
\text { dose }\end{array}$ & 21.7 & 64.7 & 10.0 & 80.0 & 50.0 \\
\hline $\begin{array}{l}\text { Volume } \\
(\mathrm{ml})\end{array}$ & Min. & 0.40 & 0.30 & 0.30 & 0.30 & 0.30 \\
\cline { 2 - 8 } & Max. & 4.00 & 4.00 & 2.50 & 2.00 & 5.00 \\
\cline { 2 - 7 } & Mean (SD) & $\begin{array}{l}1.41 \\
(0.91)\end{array}$ & $1.35(1.01)$ & $\begin{array}{l}1.53 \\
(0.82)\end{array}$ & $\begin{array}{l}0.97 \\
(0.62)\end{array}$ & $\begin{array}{l}2.23 \\
(1.57)\end{array}$ \\
\hline
\end{tabular}

